\title{
24. \\ Zur Theorie der binären kubischen Formen.
}

(Von Herrn F. Arndt.)

Der Erste, welcher Untersuchungen über die binären Formen des dritten Grades publicirt hat, ist bekanntlich Eisenstein (Bd. XXVII dieses Journals). Die Resultate derselben beschränken sich indessen auf einige wenige Theoreme, und beruhen auf derartigen Methoden, welche zur allgemeinen Lösung der Fundamentalprobleme nicht führen können. Die Bestimmung der Anzahl der Transformationen, sowie der Anzahl der kubischen Classen, welche zu einer determinirenden quadratischen Classe gehören, aber nur für den besonderen Fall, wo die Determinante dieser Classe gleich $-4 p$, und $p$ eine positive Primzahl $4 m+3$, die überdiefs zu den von Gau/s so genannten regulüren Determinanten gehört, ist im Wesentlichen Alles, was von Eisenstein in diesem Gebiete geleistet worden ist. Denn der p. 76 erweiterte und ohne Beweis aufgestellte Satz, in welchem nur noch die Einschränkung, dafs die Determinante ohne quadratischen Theiler sei, festgehalten wird, ist weder allgemein richtig (er gilt z. B. nicht für die positiven Determinanten), noch kann er durch die früheren Principien bewiesen werden.

Diese Sätze von Eisenstein waren mir noch unbekannt, als ich im Jahre 1850 an die Untersuchung der kubischen Formen ging. Die Resultate derselben sind in der Zeitschrift von Grunert Th. XVII und XIX publicirt. Man findet dort die Fundamentalprobleme, betreffend die Aequivalenz, Transformation, Eintheilung in Classen, Einschlief'sung vollständig gelöset. In Th. XIX steht der allgemeine Satz, nach welchem sich die Classification der kubischen Formén für eine beliebige Determinante bewerkstelligen läfst.

Die Auflösung einer gewissen Klasse von unbestimmten Gleichungen höherer Grade mit 3 Unbekannten hat mich nun auf eine neue, überaus einfache, Darstellung der Hauptresultate der Theorie der binären kubischen Formen geführt, die mir der Mittheilung nicht unwerth geschienen hat. Es kommt dabei ein Lemma in Anwendung, dessen Beweis ich seiner Einfachheit wegen übergehen zu dürfen glaube, nämlich: 
„Es sei $a$ prim gegen $2 b$ und $b^{2}-a c=D$; man kann eine ganze „Zahl $\boldsymbol{B}$ finden, so dafs $\boldsymbol{B} \equiv \boldsymbol{b}(\bmod \cdot \boldsymbol{a})$ und $\boldsymbol{B}^{2} \equiv \boldsymbol{D}\left(\bmod . \boldsymbol{a}^{n}\right)$ ist, wo „n eine positive ganze Zahl. Auch ist dann $a^{n}$ prim gegen $2 B$, und die "unendlich vielen Werthe von $\boldsymbol{B}$ werden nach $\operatorname{dem} \bmod . a^{n}$ congruent "sein."

"Ist ferner a prim gegen $b, b^{2}-4 a c=D$, so kann man immer den "Congruenzen $\boldsymbol{B} \equiv \boldsymbol{b}(\bmod .2 a), \boldsymbol{B}^{2} \equiv \boldsymbol{D}\left(\bmod .4 a^{n}\right)$ genügen, so dafs " $\boldsymbol{a}^{n}$ prim gegen $\boldsymbol{B}$, und die $\boldsymbol{B}$ nach dem mod. $2 a^{n}$ congruent sind."

Die Eigenschaften der kubischen Form $a x^{3}+3 b x^{2} y+3 c x y^{2}+d y^{3} \ldots(f)$ hängen gröfstentheils von der quadratischen Form

$$
2\left(b^{2}-a c\right) x^{2}+2(b c-a d) x y+2\left(c^{2}-b d\right) y^{2} \ldots(\varphi)
$$

áb, welche ich die Charakteristik genannt habe. Ihre Determinante $D=(b c-a d)^{2}-4\left(b^{2}-a c\right)\left(c^{2}-b d\right)=a^{2} d^{2}-3 b^{2} c^{2}+4 a c^{3}+4 d b^{3}-6 a b c d$ wird die Determinante von $(f)$ genannt, wobei zu bemerken, dafs $\boldsymbol{D}$ nur $\equiv 0$ oder $\equiv 1$ (mod. 4) sein kann, und dafs eine kubische Form von negativer Determinante stets eine positive quadratische Form zur Charakteristik hat. Dies folgt augenblicklich aus den beiden identischen Gleichungen:

$$
\begin{aligned}
& \left(a^{2} d+2 b^{3}-3 a b c\right)^{2}-4\left(b^{2}-a c\right)^{3}-a^{2} D=0 \\
& \left(d^{2} a+2 c^{3}-3 b c d\right)^{2}-4\left(c^{2}-b d\right)^{3}-d^{2} D=0 .
\end{aligned}
$$

Transformirt man $f$ und $\varphi$ durch dieselbe Substitution $\left(\begin{array}{cc}\alpha & \beta \\ \gamma & \delta\end{array}\right), \alpha \delta-\beta \gamma=1$ in die Formen $\left(f^{\prime}\right)$ und $\left(\varphi^{\prime}\right)$, so zeigl die Rechnung, dafs $\left(\varphi^{\prime}\right)$ wieder die Charakteristik von $\left(f^{\prime}\right)$ ist. Wenn demnach $f$, $f^{\prime}$ eigentlich aquivalent sind, so gilt dasselbe von $\varphi, \varphi^{\prime}$, welcher Satz aber nicht umgekehrt werden darf. In jedem Falle aber kommt die Frage nach der eigentlichen Aequivalens zweier kubischen Formen $f$, $f^{\prime}$ von derselben Determinante auf die Betrachtung kubischer Formen von derselben Charakteristik surück. Es sei nämlich $\varphi$ die Charakteristik von $f, \varphi^{\prime}$ die von $f^{\prime}$. Sind $\varphi, \varphi^{\prime}$ nicht eigentlich äquivalent, so gilt dasselbe von $f, f^{\prime}$. Im entgegengesetzten Falle aber transformire man $\varphi$ in $\varphi^{\prime}$ durch eine Substitution $\left(\begin{array}{ll}\alpha & \beta \\ \gamma & \delta\end{array}\right), \alpha \delta-\beta \gamma=1$, die aus der Theorie der quadratischen Formen als bekannt vorausgesetzt wer- 
den kann, und durch die nämliche Substitution $f$ in $f^{\prime \prime}$, welche letztere Form mit $f^{\prime}$ einerlei Charakteristik haben wird; da nun $f$ und $f^{\prime \prime}$ eigentlich äquivalent sind, so werden $f$ und $f^{\prime}$ eigentlich äquivalent sein, oder nicht, jenach$\operatorname{dem} f^{\prime}$ und $f^{\prime \prime}$ eigentlich äquivalent sind, oder nicht.

Um alle kubischen Formen von derselben Determinante $D=4 m$ oder $4 m+1$ zu klassificiren, stelle man zunächst die Repräsentanten aller quadratischen Klassen zur Determinante $\boldsymbol{D}$ (bei negativem $\boldsymbol{D}$ nur die positiven Formen) auf: $\varphi, \varphi^{\prime}, \varphi^{\prime \prime}$ elc., und suche die verschiedenen kubischen Formen auf, deren Charakteristiken diese letztern sind. Aequivalente quadratische Formen brauchen nämlich nicht in Betracht gezogen zu werden. Um dies zu zeigen, seien $\varphi, \psi$ eigentlich äquivalente quadratische Formen (von der Determinante $D$ ), und $f, f_{1}, f_{2}$, etc. alle kubischen Klassenrepräsentanten, deren gemeinschaftliche Charakteristik $\varphi$ ist. Man bezeichne ferner mit $g$ eine Kubikform mit der Charakteristik $\psi$, transformire $\psi$ in $\varphi$ durch die Substitution $\left(\begin{array}{ll}\alpha & \beta \\ \gamma & \delta\end{array}\right)$ wo $\alpha \delta-\beta \gamma=1$, und $g$ in eine andere Kubikform $g^{\prime}$ durch die nämliche Substitution; $g^{\prime}$ wird mit $g$ eigentlich äquivalent sein, und die Charakteristik $\varphi$ hăben. Nach der obigen Definition der Formen $f, f_{1}, f_{2}, \ldots$ ist aber $g^{\prime}$ mit irgend einer derselben eigentlich äquivalent, folglich auch $g$, wodurch die Behauptung erwiesen ist.

Die Klassification der Kubikformen von gegebener Determinante $\boldsymbol{D}$ hängt hienach von zwei Problemen ab, nämlich $1^{\circ}$. „Alle Kubikformen zu bestimmen, welche eine gegebene Charakteristik haben, und $2^{\circ}$. dieselben in Klassen zu bringen." Dabei genügt es, die primiliven kubischen Formen $(a, b, c, d)$, d. h. diejenigen zu betrachten, für welche $a, b, c, d$ ohne gemeinschaftlichen Theiler sind. Es ist hiebei wohl zu bemerken, dafs die Charakteristik einer primitiven Kubikform keine primitive quadratische Form zu sein braucht. Man hat ferner zwei Fälle zu unterscheiden, indem die Charakteristik

$$
\varphi=\left(2\left(b^{2}-a c\right), b c-a d, 2\left(c^{2}-b d\right)\right)
$$

sowohl aus einer eigentlich primitiven Form, wie aus einer uneigentlich primitiven derivirt sein kann. Im ersten Falle sei $(f, g, h)$ jene eigentlich primitive Form, ihre Determinante $=\Delta$; da $f$ und $h$ nicht beide gerade sind, so ist klar, dafs

\section{$\varphi$ die Form (2mf, 2mg, 2mh)}

haben mufs, deren Determinante $D=4 \mathrm{~m}^{2} \Delta$ ist. - Im zweiten Falle sei $(2 f, g, 2 h)$ die uneigentlich primitive Form von der Determinante $y^{2}-4 f h=A$, Journal f. d. M. Bd. LIII. Heft 4. 
wo also $y$ und $\Delta$ ungerade sind. Es erhellt, dafs hier

$\varphi$ von der Form (2mf, mg, 2mh), $D=m^{2} \Delta$ ist.

Betrachten wir nun den ersten Fall. Um alle primitiven kubischen Formen mit der Charakteristik $\varphi$ zu bestimmen, hat man den folgenden Gleichungen

$$
\text { (a.) } \quad b^{2}-a c=m f, \quad b c-a d=2 m g, \quad c^{2}-b d=m h
$$

zu genügen, so dafs die Unbekannten $a, b, c, d$ ohne gemeinschaftlichen Theiler sind. Um die Aufgabe zu vereinfachen, wollen wir annehmen, dafs $f$ prim gegen $2 m \Delta$, mithin auch prim gegen $2 g$ ist, eine statthafte Annahme, indem durch die eigentlich primitive Form $(\boldsymbol{f}, \boldsymbol{g}, \boldsymbol{h})$ unendlich viele Zahlen dargestellt werden, die gegen eine gegebene Zahl prim sind. Es wird nun behauptet, dafs unter dieser Voraussetzung $a$ und $b$ relative Primzahlen sein werden. Um dies zu zeigen, sind noch die identischen Gleichungen

$$
\text { (b.) } \quad c f-2 b g+a h=0, \quad d f-2 c g+b h=0
$$

zu beachten, welche sich aus (a.) ergeben. Es sei der gröfste gemeinschaftliche Theiler $(9)$ von $a$ und $b$ von der Einheit verschieden. Nach $(a$.) geht $\vartheta$ in $m f$ und $2 m g$, folglich in dem gröfsten gemeinsamen Maafs dieser Zahlen $(m)$ auf, ist also prim gegen $f$; daher geht $\boldsymbol{\theta}$ in $c$ auf, was aus (b.) folgt, folglich ist $m$ durch $\vartheta^{2}$ theilbar (wegen der ersten Gl. (a.)), also ad und $b d$ ebenfalls (wegen der beiden andern Gl. (i.)); nun mufs $\vartheta^{2}$ prim gegen $d$ sein, da $a, b, c, d$ keinen gemeinschaftlichen Theiler haben dürfen, folglich sind $a$ und $b$ durch $\vartheta_{2}$ theilbar, d. b. die ganzen Zahlen $\frac{a}{\boldsymbol{\vartheta}}, \frac{b}{\boldsymbol{\vartheta}}$ hätten noch den Faktor $\vartheta$ gemein, während sie relative Primzahlen sind. Da hiemit erwiesen, daf's $a$ und 1 prim gegen einander, so folgt aus (a.), dafs a prim gegen $m f$ ist.

Dies vorausgesetzt, beruht die neue Theorie auf der folgenden identischen Gleichung, die sich aus (a.) ergiebt:

(c.) $(b f-a g)^{2}-a^{2} \Delta=m f^{3}$, oder $\xi^{2}-\eta^{2} \Delta=m f^{3}$; wo $\xi=b f-a g, \eta=a$ prim gegen einander sein werden, wie leicht erhellt. Mit Hülfe dieser Gleichung lassen sich nach (a.) die Coefficienten der Kubikform durch $\xi$ und $\eta$ ausdrücken wie folgt:

(d.) $\quad a=\eta, \quad b=\frac{g \eta+\xi}{f}, \quad c=\frac{\left(y^{2}+\Delta\right) \eta+2 g \xi}{f^{2}}, \quad d=\frac{\left(g^{3}+3 g \Delta\right) \eta+\left(3 g^{2}+\Delta\right) \xi}{f^{3}}$. Man bemerke noch folgende irrationale Form, deren Anwendung. die weitere 
Betrachtung sehr vereinfacht. Bringt man den Ausdruck

$$
\frac{1}{f^{3}}(f x+g y+y \sqrt{ } \Delta)^{3}(\xi+\eta \sqrt{ } \Delta) \text { auf die Form } \varepsilon+L \sqrt{ } \Delta,
$$

so wird $L=a x^{3}+3 b x^{2} y+3 c x y^{2}+d y^{3}$, wo $a, b, c$, d die obigen Werthe haben, diejenige Kubikform, um deren Bestimmung es sich handelt. Auch die andere Form \& spielt in der Theorie der kubischen Formen eine Rolle; man kann sie die Adjuncta von $L$ nennen. Die Coefficienten dieser Form sind

$$
\mathfrak{a}=\xi, \quad \mathfrak{b}=\frac{g \xi+\Delta \eta}{f}, \quad \mathfrak{c}=\frac{\left(g^{2}+\Delta\right) \xi+2 g \Delta \eta}{f^{2}}, \quad \mathfrak{D}=\frac{\left(g^{3}+3 g \Delta\right) \xi+\left(3 g^{2}+\Delta\right) \Delta \eta}{f^{3}},
$$

und lassen sich durch $a, b, c, d, f, y, h$ wie folgt ausdrücken:

$$
\mathfrak{a}=b f-a g, \quad \mathfrak{b}=b y-a h, \quad \mathfrak{c}=d f-c g, \quad b=d g-c h .
$$

Die Form $\mathfrak{R}$ ist übrigens auch von Eisenstein bemerkt worden.

Die Zahlen $\xi$ und $\eta$ sind nun nicht willkührliche Wurzeln der unbestimmten Gleichung (c.), denn es tritt die Bedingung auf, dafs $b, c$, d ganze Zahlen seien. Zuförderst bemerken wir, dafs $c$ und $d$ von selbst ganz sein werden, wenn nur $b$ eine ganze Zahl ist. In der That aus $g \eta+\xi \equiv 0$ (mod. $f$ ) folgt $(g \eta+\xi)^{2} \equiv 0\left(\bmod . f^{2}\right)$, d. i. $\left(\left(g^{2}+\Delta\right) \eta+2 y \xi\right) \eta \equiv 0\left(\bmod . f^{2}\right) ;$ nun ist $f$ prim gegen $\eta$ (denn ein gemeinschaftlicher Theiler von $f ; \eta$ würde $\xi$ messen), folglich ist $\left(g^{2}+\mathcal{A}\right) \eta+2 g \xi \equiv 0\left(\bmod . f^{2}\right)$, d. h. $c$ eine ganze Zahl. - Es ist weiter $(g \eta+\xi)\left(\left(g^{2}+\Delta\right) \eta+2 g \xi\right) \equiv 0$ (mod. $\left.f^{3}\right)$, woraus wie vorher folgt, dafs $d$ eine ganze Zahl ist.

Wir haben also nur noch die eine Bedingung: „b ganz" zu berücksichtigen. Diese läfst sich auf eine andere zurückführen. Nach dem in der Einleitung angeführten Lemma bestimme man eine Zahl $G$ so, dafs

$$
\text { (e.) } \quad G \equiv g(\bmod . f), \quad G^{2} \equiv \Delta\left(\bmod . f^{3}\right) ;
$$

da $g \eta+\xi$ durch $f$ theilbar sein soll, so gilt dasselbe von $\boldsymbol{G} \eta+\xi$. Es ist ferner $(\xi+G \eta)(\xi-G \eta)=\left(\Delta-G^{2}\right) \eta^{2}+i n f^{3}$, also nach $(e),.(\xi+G \eta)(\xi-G \eta)$ durch $f^{3}$ theilbar. Es werden aber $f$ und $\xi-G \eta$ relative Primzahlen sein; denn ein gemeinschaftlicher Theiler dieser Zahlen würde nach dem Vorhergehenden in $\xi+G \eta$ aufgehen, mithin in $G \eta$, während doch $f$ prim gegen dieses Produkt ist. Hieraus folgt $\xi+G \eta \equiv 0$ (mod. $f^{3}$ ). Man setze die Zahl links $=f^{3} x$, und substituire den sich ergebenden Werth $\xi$ in (c.); es kommt die Gleichung

$$
\text { (f.) } f^{3} \tau^{2}-2 G \tau \eta+\frac{G^{2}-\Delta}{f^{3}} \eta^{2}=m \text {, }
$$

und wenn man alle möglichen Werthe $\tau, \eta$ bestimmt, welche dieser Gleichung 
genügen und prim gegen einander sind, ferner die entsprechenden $\xi=f^{3} \tau-G \eta$ berechnet, so hat man nach $(d$.) alle Kubikformen $(a, b, c, d)$, welche die Form $\varphi=(2 m f, 2 m g, 2 m h)$ zur Charakteristik haben. Die Aufgabe ist hienach darauf zurückgeführt, alle Darstellungen der Zahl $m$ durch die quadratische Form

$$
\varphi \ldots\left(f^{3},-G, \frac{G^{2}-\Delta}{t^{3}}\right)
$$

von der Determinante $\Delta$ zu bestimmen. Es ist übrigens leicht zu sehen, dafs diese Form durch Triplication der Form $(f, g, h)$ von der nämlichen Determinante entsteht.

Die erste Bedingung der Lösbarkeit der Gleichung ( $f$.) besteht darin, dafs $\Delta$ quadratischer Rest von $m$ ist. Ist sie erfüllt, so vertheilen sich nach Gau/s die sämmtlichen Lösungen in eine endliche Anzahl von Gruppen, die zu den verschiedenen Werthen des Ausdrucks $\omega \equiv \sqrt{ } \Delta$ (mod.m) gehören. Für ein bestimmtes $\omega$ entspricht eine Gruppe von Auflösungen den sämmtlichen eigentlichen Transformationen der Form $\varphi$ in die Form

$$
\left(m, \omega, \frac{\omega^{2}-\Delta}{m}\right) \text {, }
$$

mit der sie eigentlich aequivalent sein mufs. Bezeichnet man ferner mit $\tau_{0}, \eta_{0}$ eine individuelle Lösung der Gruppe, so erhält man bekanntlich alle übrigen Lösungen dieser Gruppe $\tau$, $\eta$ durch die Formeln

$$
\boldsymbol{\tau}=\boldsymbol{\tau}_{0} \boldsymbol{T}+\left(\boldsymbol{\tau}_{0} \boldsymbol{G}-\eta_{0} \boldsymbol{H}\right) \boldsymbol{U}, \quad \eta=\eta_{0} \boldsymbol{T}+\left(\boldsymbol{\tau}_{0} \boldsymbol{f}^{3}-\eta_{0} \boldsymbol{G}\right) \boldsymbol{U},
$$

wo $H=\frac{G^{2}-\Delta}{f^{3}}$ und $T^{2}-\Delta U^{2}=1$ ist. Setzt man noch $\dot{\xi}_{0}=f^{3} x_{0}-G \eta_{0}$, so findet sich leicht $\xi+\eta \sqrt{ } \Delta=\left(\xi_{0}+\eta_{0} \sqrt{ } \Delta\right)(\boldsymbol{T}+\boldsymbol{U} \sqrt{ } \Delta)$. Die kubische Form $\boldsymbol{L}_{0}$, welche der individuellen Lösung entspricht, ist durch die Gleichung

$$
\frac{1}{f^{3}}(f x+g y+y \sqrt{ } \Delta)^{3}\left(\xi_{0}+\eta_{0} \sqrt{ } \Delta\right)=\Omega_{0}+L_{0} \sqrt{ } \Delta
$$

bestimmt, jede andere durch eine ähnliche Gleichung, in welcher $\xi, \eta, \mathfrak{\&}, \boldsymbol{L}$ statt $\xi_{0}, \eta_{0}, \mathfrak{R}_{0}, \boldsymbol{L}_{0}$ stehen. Nach dem Vorhergehenden ist also

$$
\text { (g.) } \boldsymbol{s}+\boldsymbol{L} \sqrt{ } \Delta=\left(\boldsymbol{\Omega}_{0}+\boldsymbol{L}_{0} \sqrt{ } \Delta\right)(\boldsymbol{T}+\boldsymbol{U} \sqrt{ } \Delta) \text {. }
$$

Setzt man, um $L$ zu entwickeln, $L_{0}=\left(a_{0}, b_{0}, c_{0}, d_{0}\right)$, mithin

$$
\mathfrak{\Re}_{0}=\left(b_{0} f-a_{0} g, b_{0} g-a_{0} h, d_{0} f-c_{0} g, d_{0} g-c_{0} h\right),
$$

ferner $L=(a, b, c, d)$, so kommt 
Wir haben somit folgenden Satz erwiesen:

$$
\text { (h.) }\left\{\begin{array}{l}
a=a_{0} \boldsymbol{T}+\left(b_{0} f-a_{0} g\right) U \\
b=b_{0} T+\left(b_{0} g-a_{0} h\right) U \\
c=c_{0} \boldsymbol{T}+\left(d_{0} f-c_{0} g\right) U \\
d=d_{0} \boldsymbol{T}+\left(d_{0} g-c_{0} h\right) U
\end{array}\right.
$$

\section{Theorem.}

„Es sei $(f, g, h)$ eine eigentlich primitive Form von der Determinante $\Delta$, und $f$ prim gegen $2 m \Delta$, ferner $G \equiv g(\bmod . f), G^{2} \equiv \Delta\left(\bmod . f^{3}\right)$. Die sämmtlichen primitiven Kubikformen, welche die gemeinschaftliche Charakteristik $\varphi=(2 m f, 2 m g, 2 m /)$ haben, vertheilen sich in eine endliche Anzahl von Gruppen, die den verschiedenen Wurzeln der Congruenz $\omega^{2} \equiv \Delta$ (mod. $m$ ) entsprechen. Aus einer individuellen Form jeder Gruppe $\left(a_{0}, b_{0}, c_{0}, d_{0}\right)$ findet man alle übrigen Formen der nämlichen Gruppe $(a, b, c, d)$ nach den Formeln $(h$.), während die Coefficienten der individuellen Form folgende Werthe haben:

$$
\begin{gathered}
\boldsymbol{a}_{0}=\eta_{0}, \quad \boldsymbol{b}_{0}=\frac{g \eta_{0}+\xi_{0}}{f}, \quad c_{0}=\frac{\left(g^{2}+\Delta\right) \eta_{0}+2 g \xi_{0}}{f^{2}}, \\
d_{0}=\frac{\left(g^{3}+3 g \Delta\right) \eta_{0}+\left(3 g^{2}+\Delta\right) \xi_{0}}{f^{3}} .
\end{gathered}
$$

Hier ist $\xi_{0}=f^{3} \tau_{0}-G \eta_{0}$, und $\tau_{1}, \eta_{0}$ sind beliebige Wurzeln der unbestimmten Gleichung

$$
f^{3} \tau^{2}-2 G \tau \eta+\frac{G^{2}-\Delta}{f^{3}} \eta^{2}=m,
$$

prim gegen einander und aus derjenigen Gruppe von Lösungen, die man betrachtet, die also einem bestimmten Werth von $\omega \equiv \sqrt{\Delta}$ (mod. in) entsprechen."

Wir haben uns nun mit der Klassification der Formen $(f)(a, b, c, d)$ zu beschäftigen. Nach dem Obigen kann man

$$
\frac{1}{f^{3}}(f x+g y+y \sqrt{ } \Delta)^{3}(\xi+\eta \sqrt{ } \Delta)=\mathfrak{Q}+\boldsymbol{L} \sqrt{ } \Delta
$$

setzen, so dafs $f=L$ ist, wo $\xi, \eta$ ihre obige Bedeutung behalten. Man erhält alle mit $\boldsymbol{L}$ eigentlich äquivalente Formen von derselben Charakteristik $\varphi$, wenn man $\boldsymbol{L}$ durch alle diejenigen eigentlichen Substitutionen transformirt, durch welche $\varphi$ in sich selbst übergeht. Durch diese Substitutionen geht offen- 
bar auch die Form $(f, g, h)$ in sich selbst über; die Substitution ist also

$$
\boldsymbol{x}=(\boldsymbol{T}-\boldsymbol{g} \boldsymbol{U}) \boldsymbol{x}^{\prime}-\boldsymbol{h} \boldsymbol{U} \boldsymbol{y}^{\prime}, \quad \boldsymbol{y}=\boldsymbol{f} \boldsymbol{U} \boldsymbol{x}^{\prime}+\left(\boldsymbol{T}+\boldsymbol{g} \boldsymbol{U} ; \boldsymbol{y}^{\prime},\right.
$$

wo $\boldsymbol{T}, \boldsymbol{U}$ ihre obige Bedeutung beibehalten. Hienach findet sich

$$
f x+g y+y \sqrt{ } \Delta=\left(f x^{\prime}+g y^{\prime}+y^{\prime} \sqrt{ } \Delta\right)(\boldsymbol{T}+U \sqrt{ } \Delta),
$$

folglich geht $\&+L \sqrt{ } \Delta$ über in $\frac{1}{f^{3}}(f x+g y+y \sqrt{ } \Delta)^{3}(\xi+\eta \sqrt{ } \Delta)(T+U \sqrt{ } \Delta)^{3}$, d. h. in $\left(\mathcal{R}+L_{V} \Delta\right)\left(\boldsymbol{T}+U_{V} / \Delta\right)^{3}$. Wir haben also folgenden Satz bewiesen:

\section{Theorem.}

„Es sei $\boldsymbol{f} \ldots(\boldsymbol{a}, \boldsymbol{b}, \boldsymbol{c}, \boldsymbol{d})$ eine Kubikform mit der Charakteristik $\varphi \ldots(2 m f, 2 m g, 2 m h)$, wo $(f, g, h)$ eine eigentlich primitive Form von der Determinante $\boldsymbol{A}$. Man findet alle mit $\boldsymbol{f}$ eigentlich äquivalente kubische Formen mit der nämlichen Charakteristik $\varphi$, nämlich $f^{\prime} \ldots\left(a^{\prime}, b^{\prime}, c^{\prime}, d^{\prime}\right)$ nach folgenden Formeln:

$$
\begin{aligned}
\boldsymbol{a}^{\prime} & =\boldsymbol{a} \boldsymbol{T}^{\prime}+(\boldsymbol{b} f-\boldsymbol{a} \boldsymbol{g}) \boldsymbol{U}^{\prime} \\
\boldsymbol{b}^{\prime} & =\boldsymbol{b} \boldsymbol{T}^{\prime \prime}+(\boldsymbol{b} \boldsymbol{g}-\boldsymbol{a h}) \boldsymbol{U}^{\prime} \\
\boldsymbol{c}^{\prime} & =\boldsymbol{c} \boldsymbol{T}^{\prime}+(\boldsymbol{d} f-\boldsymbol{c} \boldsymbol{g}) \boldsymbol{U}^{\prime} \\
\boldsymbol{d}^{\prime} & =\boldsymbol{d} \boldsymbol{T}^{\prime}+(\boldsymbol{d} \boldsymbol{g}-\boldsymbol{c h}) \boldsymbol{U}^{\prime},
\end{aligned}
$$

wo $\boldsymbol{T}^{\prime \prime}+\boldsymbol{U}^{\prime} \sqrt{ } \Delta=(\boldsymbol{T}+\boldsymbol{U} \sqrt{ } \Delta)^{3}$, und $\boldsymbol{T}, \boldsymbol{U}$ alle möglichen Wurzein der unbestimmten Gleichung $\boldsymbol{T}^{2}-\Delta \boldsymbol{U}^{2}=1$ sind."

Vergleicht man diese Formeln mit (h.), so ergiebt sich, dafs eigentlich äquivalente Kubikformen mit derselben Charakteristik nothwendig derselben Gruppe anyehören, so dafs also Formen aus verschiedenen Gruppen auch verschiedenen Klassen angehören.

Um nun die Formen derselben Gruppe zu klassificiren, unterscheiden wir folgende Fälle:

$1^{\circ}$. Ist $\Delta$ ein Quadrat, so enthält die Gruppe (h.) nur zwei Formen $\left(a_{10}, b_{10}, c_{10}, d_{10}\right)$ und $\left(-a_{10},-b_{0},-c_{10},-d_{0}\right)$, da die Gleichung $\boldsymbol{T} \boldsymbol{T}-\Delta \boldsymbol{U} \boldsymbol{U}=1$ in diesem Falle nur die beiden Wurzelpaare $\boldsymbol{T}= \pm 1, \boldsymbol{U}=0$ zuläfst. Diese Formen bilden eine Klasse, da die eine in die andere durch die Substitution $\left(\begin{array}{rr}-1, & 0 \\ 0, & -1\end{array}\right)$ übergeht.

$2^{\circ}$. Ist $\Delta$ negativ, so gilt dasselbe. In dem Falle $\Delta=-1$ jedoch kommt noch die Lösung $\boldsymbol{T}=0, \boldsymbol{U}= \pm 1$ hinzu, aber die 4 Formen, welche die Gruppe nun enthält, constituiren wiederum eine einzige Klasse, denn für 
$T=0, U= \pm 1$ kommt $T^{\prime}=0, U^{\prime}= \pm 1$, weshalb die betreffenden Formen nach dem vorhergehenden Theorem eigentlich äquivalent sind.

$3^{\circ}$. Ist $\Delta$ endlich positiv, so läfst sich erweisen, dafs die unendlich vielen Formen derselben Gruppe drei verschiedene Klassen constituiren.

Um dies zu zeigen, werfe man aus (h.) zunächst alle die Formen aus, welche durch Veränderung aller Vorzeichen anderer Formen entstehen, was darauf hinauskommt, $\boldsymbol{T}$ als positiv zu betrachten. Sind nun $t, u$ die kleinsten positiven Wurzeln der Gleichung $\boldsymbol{T} \boldsymbol{T}-\Delta \boldsymbol{U} \boldsymbol{U}=1$, verschieden von $\boldsymbol{T}=1$, $U=0$, so erhält man bekanntlich alle übrigen Wurzeln durch die Formel

$$
\boldsymbol{T}+\boldsymbol{U} \boldsymbol{\Delta}=(\boldsymbol{t}+\boldsymbol{u} \sqrt{ } \boldsymbol{\Delta})^{\lambda},
$$

wo $\lambda$ jede beliebige ganze Zahl zwischen $-\infty$ und $+\infty$ bezeichnet. Bedeutet nun $\boldsymbol{L}_{0}$ die Grundform der Gruppe, während $\boldsymbol{L}, \boldsymbol{L}^{\prime}$ zwei andere Formen derselben, so hat man

$$
\begin{array}{ll}
\boldsymbol{L}=\boldsymbol{L}_{0} \boldsymbol{T}+\mathfrak{\Omega}_{0} \boldsymbol{U}, & \mathfrak{\ell}=\mathfrak{\Omega}_{0} \boldsymbol{T}+\boldsymbol{L}_{0} \Delta \boldsymbol{U}, \\
\boldsymbol{L}^{\prime}=\boldsymbol{L}_{0} \boldsymbol{T}^{\prime}+\mathfrak{s}_{11} \boldsymbol{U}^{\prime}, & \mathfrak{\Omega}^{\prime}=\mathfrak{\Omega}_{11} \boldsymbol{T}^{\prime \prime}+\boldsymbol{L}_{0} \Delta \boldsymbol{U}^{\prime},
\end{array}
$$

und hieraus folgt

$$
\boldsymbol{L} \boldsymbol{T}^{\prime}+\mathfrak{s} \boldsymbol{U}^{\prime}=\boldsymbol{L}^{\prime} \boldsymbol{T}+\boldsymbol{\ell}^{\prime} \boldsymbol{U}, \quad \boldsymbol{\Omega}^{\prime} \boldsymbol{T}^{\prime}+\boldsymbol{L} \Delta \boldsymbol{U}^{\prime}=\mathfrak{\ell}^{\prime} \boldsymbol{T}+\boldsymbol{L}^{\prime} \boldsymbol{\Delta} \boldsymbol{U},
$$

also durch Elimination von $\mathfrak{\Omega}^{\prime}$ :

$$
\boldsymbol{L}^{\prime}=\boldsymbol{L}\left(\boldsymbol{T} \boldsymbol{T}^{\prime}-\boldsymbol{\Delta} \boldsymbol{U} \boldsymbol{U}^{\prime}\right)+\boldsymbol{\ell}\left(\boldsymbol{T} \boldsymbol{U}^{\prime}-\boldsymbol{U} \boldsymbol{T}^{\prime}\right),
$$

d. h. wenn die Formen $(a, b, c, d) ;\left(a^{\prime}, b^{\prime}, c^{\prime}, d^{\prime}\right)$ den Werthen $T, U ; T^{\prime}, U^{\prime}$ ihrer Gruppe entsprechen, so kann man die eine Form durch die andere so ausdrücken :

$$
\begin{aligned}
\boldsymbol{a}^{\prime} & =\boldsymbol{a} \boldsymbol{T}^{\prime \prime}+(\boldsymbol{b} f-\boldsymbol{a} \boldsymbol{g}) \boldsymbol{U}^{\prime \prime} \\
\boldsymbol{b}^{\prime} & =\boldsymbol{b} \boldsymbol{T}^{\prime \prime}+(\boldsymbol{b} \boldsymbol{g}-\boldsymbol{a} \boldsymbol{h}) \boldsymbol{U}^{\prime \prime} \\
\boldsymbol{c}^{\prime} & =\boldsymbol{c} \boldsymbol{T}^{\prime \prime}+(\boldsymbol{d} \boldsymbol{f}-\boldsymbol{c} \boldsymbol{g}) \boldsymbol{U}^{\prime \prime} \\
\boldsymbol{d}^{\prime} & =\boldsymbol{d} \boldsymbol{T}^{\prime \prime}+(\boldsymbol{d} \boldsymbol{g}-\boldsymbol{c h}) \boldsymbol{U}^{\prime \prime},
\end{aligned}
$$

so dafs $\boldsymbol{T}^{\prime \prime}=\boldsymbol{T} \boldsymbol{T}^{\prime}-\Delta \boldsymbol{U} \boldsymbol{U}^{\prime}$ und $\boldsymbol{U}^{\prime \prime}=\boldsymbol{T} \boldsymbol{U}^{\prime}-\boldsymbol{U} \boldsymbol{T}^{\prime \prime}$ ist. Macht man nun

$$
\boldsymbol{T}+\boldsymbol{U}^{\prime} \boldsymbol{\Delta}=\left(\boldsymbol{t}+u_{\sqrt{ }} \Delta\right)^{\lambda}, \quad \boldsymbol{T}^{\prime}+\boldsymbol{U}^{\prime} \sqrt{\Delta}=\left(\boldsymbol{t}+\boldsymbol{u}_{\sqrt{ } \boldsymbol{\Delta}}\right)^{\lambda^{\prime},},
$$

so kommt

$$
\boldsymbol{T}^{\prime \prime}+\boldsymbol{U}^{\prime \prime} \sqrt{\Delta}=(\boldsymbol{t}+\boldsymbol{u} \sqrt{\Delta})^{\lambda^{\prime}-\lambda} .
$$

Sollen die betrachteten Formen eigentlich äquivalent sein, so mufs nach dem obigen Theorem $\boldsymbol{T}^{\prime \prime}+\boldsymbol{U}^{\prime \prime} \sqrt{ } \boldsymbol{\Delta}$ die Form $(t+u \sqrt{\Delta})^{3 \mu}$ haben, und umgekehrt, also kommt $\lambda^{\prime}-\lambda=3 \mu$. Hieraus ersieht man leicht, dafs die Formen der- 
selben Gruppe in der That 3 Klassen constituiren; die Repräsentanten derselben erhält man aus (h.), wenn man $\boldsymbol{T}+\boldsymbol{U} V \Delta=(t+u \sqrt{ } \Delta)^{\lambda}$ setzt, und für $\lambda$ irgend drei nach dem mod. 3 incongruente Werthe nimmt, z. B. $0,1,2$.

\section{Theorem.}

"Alle Kubikformen mit derselben Charakteristik (2mf, 2mg, 2mh), die eine Gruppe bilden, constituiren drei Klassen, wenn $\Delta=g^{2}-f^{\prime} h$ positiv und nicht quadratisch ist, in den übrigen Fällen eine Klasse."

Wir kommen nun zu dem zweiten Hauptfalle. Die aufzulösenden Gleichungen sind hier

$$
b^{2}-a c=m f, \quad b c-a d=m g, \quad c^{2}-b d=m h .
$$

Man darf $f$ prim gegen $2 m \Delta$ nehmen; dafs alsdann $f$ prim gegen $2 g$, $a$ und $b$ prim gegen einander, und $a$ prim gegen $m f$, wird wie oben bewiesen. Statt der Gleichung (c.) kommt

$$
\xi^{2}-\eta^{2} \Delta=4 m f^{3}, \quad \xi=2 b f-a g, \quad \eta=a .
$$

$\xi$ und $\eta$ werden keinen ungeraden Faktor gemein haben, und entweder beide gerade, oder beide ungerade sein, wie leicht erhellt. Für die Coeficienten der Kubikform erhält man die Werthe:

$$
\begin{array}{rlrl}
a=\eta, & b & =\frac{g \eta+\xi}{2 f}, \quad c=\frac{\left(g^{2}+\Delta\right) \eta+2 g \xi}{4 f^{2}}, \\
d & =\frac{\left(g^{3}+3 g \Delta\right) \eta+\left(3 g^{2}+\Delta\right) \xi}{8 f^{3}},
\end{array}
$$

oder auch, wenn die Form mit $\boldsymbol{L}$ bezeichnet wird, so ist

$$
\frac{1}{8 f^{3}}(2 f x+g y+y \sqrt{ } \Delta)^{3}(\xi+\eta \sqrt{ } \Delta)=\mathfrak{s}+\boldsymbol{L} \sqrt{ } \Delta .
$$

Ist $b$ ganz, so werden $c, d$ ebenfalls ganze Zahlen sein. Denn

$$
\left(2 g \xi+\left(g^{2}+\Delta\right) \eta\right) \eta \equiv 0 \quad\left(\bmod .4 f^{2}\right)
$$

wenn also $\eta$ ungerade, so ist $c$ eine ganze Zahl, wenn aber $\eta$ gerade, so ist der Faktor von $\eta$ links sowohl durch 4 , als auch durch $f^{2}$ theilbar, mithin durch $4 f^{2}$, also $c$ wieder ganz. - Ferner

$$
\left(\left(g^{3}+3 g \Delta\right) \eta+\left(3 g^{2}+\Delta\right) \xi\right) \eta \equiv 0 \quad\left(\bmod .8 f^{3}\right),
$$

also $d$ ganz, wenn $\eta$ ungerade; wenn aber $\eta$ gerade, so folgt dasselbe, da der Faktor von $\eta$ links dann durch 8 theilbar ist. 
Nach dem Lemma der Einleitung kann man $\boldsymbol{G}$ so bestimmen, dafs $\boldsymbol{G} \equiv \boldsymbol{g}(\bmod .2 f), \boldsymbol{G}^{2} \equiv \Delta\left(\bmod .4 f^{3}\right)$ ist, und $\xi+G \eta$ mufs durch $2 f$ theilbar sein. Man hat ferner $(\xi+G \eta)(\xi-G \eta) \equiv\left(\Delta-G^{2}\right) \eta^{2} \equiv 0\left(\bmod .4 f^{3}\right)$. Ist nun erstens $\eta$ gerade, so folgt leicht, dafs $f$ prim gegen $\xi-G \eta$, also $\xi+G \eta$ durch $f^{3}$ theilbar ist; die letzte Zahl ist aber ungerade, folglich $\xi+G \eta$ durch $2 f^{3}$ theilbar. In diesem Falle ist $\left(\frac{\xi}{2}\right)^{2}-\Delta\left(\frac{\eta}{2}\right)^{2}=m f^{3}$ ungerade, folglich $\frac{\xi}{2}+G \frac{\eta}{2}$ ungerade. Setzen wir also $\xi+G \eta=2 \gamma^{3} \tau$, so ist $\tau$ ungerade, mithin prim gegen $\eta$. - Ist zweitens $\eta$ ungerade, so kommt

$$
\frac{1}{2}(\xi+G \eta) \cdot \frac{1}{2}(\xi-G \eta) \equiv 0 \quad\left(\bmod . f^{3}\right),
$$

und da leicht zu zeigen, dafs $f$ prim gegen den zweiten Faktor, so kommt $\frac{1}{2}(\xi+G \eta) \equiv 0\left(\bmod . f^{3}\right)$, also wie vorher $\xi+G \eta=2 f^{3} \tau$, so dafs $\tau$ prim gegen $\eta$ ist. Statt der Formel (f.) erhält man so die folgende:

$$
2 f^{3} \tau^{2}-2 G \tau \eta+2 \frac{G^{2}-\Delta}{4 f^{3}} \eta^{2}=2 m, \quad \xi=2 f^{3} \tau-G \eta
$$

wobei zu bemerken, dafs

$$
\left(2 f^{3},-G, 2 \frac{G^{2}-\Delta}{4 f^{3}}\right)
$$

eine uneigentlich primitive Form von der Determinante $\Delta$ ist. Die Coefficienten der Form $\&$ werden

$$
\mathfrak{a}=2 b f-a g, \quad \mathfrak{b}=b y-2 a h, \quad \mathfrak{c}=2 d f-c g, \quad \mathfrak{b}=d g-2 c h .
$$

Das dem ersten Theorem entsprechende lautet nun wie folgt:

Theorem.

„Es sei $(2 f, g, 2 h)$ eine uneigentlich primitive Form von der Determinante $\Delta$, und $f$ prim gegen $2 m \Delta$, ferner $G \equiv g$ (mod. $2 f), G^{2} \equiv A$ $\left(\bmod .4 f^{3}\right)$. Die sämmtlichen primitiven Kubikformen, welche die gemeinschaftliche Charakteristik $\varphi=(2 m f, m g, 2 m h)$ haben, vertheilen sich in eine endliche Anzahl von Gruppen, die den verschiedenen Wurzeln der Congruenz $\omega^{2} \equiv \Delta(\bmod .2 m)$ entsprechen. Aus einer individuellen Form jeder Gruppe $\left(a_{0}, b_{0}, c_{0}, d_{0}\right)$ findet man alle übrigen Formen der nämlichen Gruppe $(a, b, c, d)$ nach den Formeln:

$$
\left(h^{\prime} .\right) \quad\left\{\begin{array}{l}
2 a=a_{0} T+\left(2 b_{0} f-a_{0} g\right) U \\
2 b=b_{0} T+\left(b_{0} g-2 a_{0} h\right) U \\
2 c=c_{0} T+\left(2 d_{0} f-c_{0} g\right) U \\
2 d=d_{0} T+\left(d_{0} g-2 c_{0} h\right) U,
\end{array}\right.
$$

Journal f. d. M. Bd. Lill. Heft 4. 
wo $\boldsymbol{T}, \boldsymbol{U}$ alle Wurzeln der unbestimmten Gleichung $\boldsymbol{T}^{2}-\Delta \boldsymbol{U}^{2}=4$ bedeuten, während die Coefficienten der individuellen Form folgende Werthe haben:

$$
\begin{aligned}
\boldsymbol{a}_{0}=\eta_{10}, \quad \boldsymbol{b}_{0} & =\frac{g \eta_{0}+\xi_{0}}{2 f}, \quad \boldsymbol{c}_{0}=\frac{\left(g^{2}+\Delta\right) \eta_{0}+2 g \xi_{0}}{4 f^{2}}, \\
\boldsymbol{d}_{0} & =\frac{\left(g^{3}+3 g \Delta\right) \eta_{0}+\left(3 g^{2}+\Delta\right) \xi_{0}}{8 f^{3}} .
\end{aligned}
$$

Hier ist $\xi_{0}=2 f^{3} \tau_{0}-G \eta_{0}$, und $\tau_{0}, \eta_{0}$ sind beliebige Wurzeln der unbestimmten Gleichung

$$
2 f^{3} \tau^{2}-2 G \tau \eta+2 \frac{G^{2}-\Delta}{4 f^{3}} \eta^{2}=2 m,
$$

prim gegen einander und aus derjenigen Gruppe von Lösungen, die man betrachtet, die also einem bestimmten Werth von $\omega \equiv \sqrt{ } \Delta(\bmod .2 m)$ entsprechen."

Da ferner die Form $(2 f, g, 2 h)$ in sich selbst übergeht durch die eigentlichen Transformationen

$$
x=\frac{1}{2}(\boldsymbol{T}-\boldsymbol{g} \boldsymbol{U}) \boldsymbol{x}^{\prime}-\boldsymbol{h} \boldsymbol{U} \boldsymbol{y}^{\prime}, \quad y=\boldsymbol{f} \boldsymbol{U} \boldsymbol{x}^{\prime}+\frac{1}{2}(\boldsymbol{T}+\boldsymbol{g} \boldsymbol{U}) \boldsymbol{y}^{\prime},
$$

so gelangt man ähnlich wie Oben zu dem folgenden

\section{Theorem.}

„Es sei $f \ldots(a, b, c, d)$ eine Kubikform mit der Charakteristik $\varphi \ldots(2 m f, m g, 2 m h)$, wo $(2 f, g, 2 h)$ eine uneigentlich primitive Form von der Determinante $\Delta$. Man findet alle mit $f$ eigentlich äquivalente kubische Formen mit der nämlichen Charakteristik $\varphi$, nämlich $f^{\prime} \ldots\left(a^{\prime}, b^{\prime}, c^{\prime}, d^{\prime}\right)$ nach folgenden Formeln:

$$
\begin{aligned}
& 2 a^{\prime}=a \boldsymbol{T}^{\prime}+(2 b f-a g) \boldsymbol{U}^{\prime} \\
& 2 b^{\prime}=b \boldsymbol{T}^{\prime}+(b g-2 a h) \boldsymbol{U}^{\prime} \\
& 2 c^{\prime}=c \boldsymbol{T}^{\prime}+(2 d f-c g) \boldsymbol{U}^{\prime} \\
& 2 d^{\prime}=d \boldsymbol{T}^{\prime}+(d g-2 c h) \boldsymbol{U}^{\prime}
\end{aligned}
$$

wo $\boldsymbol{T}^{\prime}+U^{\prime} \sqrt{\Delta}=2\left(\frac{T}{2}+\frac{U}{2} \sqrt{\Delta}\right)^{3}$, und $\boldsymbol{T}, \boldsymbol{U}$ alle möglichen Wurzeln der unbestimmten Gleichung $\boldsymbol{T} \boldsymbol{T}-\Delta \boldsymbol{U} \boldsymbol{U}=4$ sind."

Um die Formen derselben Gruppe zu klassificiren, sei

1 $^{\circ}$ A ein Quadrat. Dann hat die Gruppe nur zwei Formen, die in eine Klasse gehören.

Ist $2^{\circ} . \Delta$ negativ ${ }^{*}$ ), so ist $\boldsymbol{T}=2, U=0^{* *}$ ) für ein beliebiges $\Delta$;

*) Man bemerke dafs $\Delta$ nicht $=-1$ sein kann, wegen $g^{2}-4 f h=\Delta$.

**) Die Formen, welche durch Veränderung aller Vorzeichen andrer Formen entstehen, kann man aus der Gruppe auswerfen. 
$\boldsymbol{T}=1, \boldsymbol{U}= \pm 1$ für $\Delta=-3$. Ist nun $\Delta$ nicht $=-3$, so hat die Gruppe nur eine Form. Ist $\Delta=-3$, so hat sie 3 Formen, welche auch 3 Klassen constituiren, da die Bedingung $1 \pm \Delta=2(1+0 . \Delta)^{3}$ nicht möglich ist.

$3^{\circ}$. Ist $\Delta$ positiv, so findet sich wie Oben, dafs die Formen der Gruppe wiederum 3 Klassen bilden, deren Repräsentanten man aus $\left(h^{\prime}\right.$.) erhält, wenn man

$$
T+U \sqrt{ } \Delta=2\left(\frac{t}{2}+\frac{u}{2} \sqrt{ } \Delta\right)^{\lambda}
$$

setzt, und für $\lambda$ irgend drei nach dem mod. 3 incongruente Werthe nimmt, z. B. $0,1,2$. Hier sind $t, u$ die kleinsten positiven Wurzeln der Gleichung

$$
\boldsymbol{T}^{2}-\Delta \boldsymbol{U}^{2}=4
$$

verschieden von $T=2, U=0$.

\section{Theorein.}

„Alle Kubikformen von derselben Charakteristik (2mf, $m g, 2 m h$ ), die eine Gruppe bilden, constituiren drei Klassen, wenn $\Delta=g^{2}-4$ fh positiv und nicht quadratisch, und wenn $\Delta=-3$ ist, in den übrigen Fällen eine Klasse."

Da nun die Anzahl der quadratischen Klassen für eine gegebene Determinante endlich ist ${ }^{*}$ ), und da zu jeder solchen Klasse eine endliche Anzahl von kubischen Klassen gehört, so gelangt man zu dem

\section{Fundamentalsats.}

„Alle Kubikformen von derselben nicht verschwindenden Determinante $^{* *}$ ) zerfallen in eine endliche Menge verschiedener Klassen."

*) Formen von der Determinante Null ausgeschlossen.

**) Wenn die Determinante Null, so ist die Anzahl der Klassen unendlich grofs.

Berlin, den 6. September 1855. 